\title{
PRODUCCIÓN Y PERCEPCIÓN DE BIENES PÚBLICOS EN LA LÓGICA DE LA ACCIÓN COLECTIVA
}

\author{
THE LOGIC OF COLLECTIVE ACTION. THE ROLE OF PUBLIC GOODS \\ PRODUCTION AND PERCEPTION
}

\author{
JACINT JORDANA \\ Universitat Pompeu Fabra e Instituto de Estudios Internacionales de Barcelona. España \\ jacint.jordana@upf.edu
}

\begin{abstract}
RESUMEN
Bienes públicos con distintas propiedades generan problemas diferentes de acción colectiva. Esta hipótesis ha sido discutida, desde distintas perspectivas analíticas, con el objeto de profundizar teóricamente sobre la lógica de la acción colectiva. En este trabajo se revisan las aportaciones recientes a esta cuestión, analizando sus puntos de encuentro y sus divergencias en cuanto a conceptos y resultados esperados. Asimismo, se examina la relación entre tipos distintos de bienes públicos y el comportamiento interdependiente de los individuos. Con este fin, los vínculos entre las funciones de producción y las formas de consumo de bienes públicos también son considerados, ya que permite desarrollar modelos más ambiciosos sobre la lógica de la acción colectiva. Finalmente, se considera la existencia de posibles problemas de percepción en los individuos que participan en la acción colectiva, produciendo desviaciones sistemáticas en la racionalidad de las decisiones, lo que nos permite concluir que, en lugar de pretender una teoría que incluya todos los problemas de la acción colectiva, deberíamos identificar patrones sistemáticos de comportamiento, susceptibles de ser contrastados empíricamente.
\end{abstract}

\section{Palabras Clave Adicionales}

Teoria de Juegos, Grupos de Intereses, Dilema del Prisionero, Bienes Comunales, Bienes Colectivos, Oferta conjunta.

\begin{abstract}
Public goods having different proprieties produce different problems of collective action. This hypothesis has been discussed from different analytical perspectives and we examine their arguments, aiming to deepen our theoretical understanding about the logic of collective action. In this paper we look for similarities and differences regarding their use of concepts and expected results. Simultaneously, we examine the link between different public goods and the interdependent behavior of individuals. To this purpose, we also revisit the connections between production functions and consumption types in the case of public goods, as a mean to explore the potential development of more elaborated models in the theory of collective action, and suggest that more empirical contrast of these models is needed. Finally, we argue the importance for theoretical discussions to include a discussion about the impact of perception in the collective action models, considering systematic deviations to rational choice, and conclude that no single theory may address all collective action problems, but systematic patterns of behavior may be identified and empirically confirmed.
\end{abstract}

\section{Additional KeYWords}

Game Theory, Interest Groups, Prisoner's Dilemma, Collective Goods, Common Goods, Jointness of Supply. 


\section{INTRODUCCIÓN}

El desarrollo de una teoría de la acción colectiva en las ciencias sociales no ha sido demasiado afortunado en las últimas décadas. En los años sesenta, la contribución seminal de Mancur Olson, The Logic of Collective Action (1965), estableció una base conceptual innovadora para pensar la relación entre intereses individuales e intereses de grupo (o colectivos) y planteó como problema la participación en una acción colectiva para defender intereses comunes. Sin embargo, los trabajos posteriores no alcanzaron a establecer una base analítica común suficientemente robusta y sofisticada, que mostrase un desarrollo teórico sostenido e integrado. Ello no quiere decir que la perspectiva analítica aportada por "la lógica de la acción colectiva" no haya sido impulsora de fructíferas contribuciones al análisis social en las últimas décadas, o que estimulara los debates sobre la aplicación de la hipótesis de la racionalidad al estudio de la acción colectiva.

En efecto, el impacto de la obra de Mancur Olson en las ciencias sociales ha sido muy elevado, como lo muestra el enorme número de libros y artículos publicados desde entonces, con variedad de perspectivas, sobre las ideas contenidas en el libro. Sin entrar a caracterizar los debates teóricos, es posible señalar que rápidamente surgió una importante línea de investigación que tradujo la lógica de la acción colectiva al campo de la teoría de juegos, proponiendo el modelo del "dilema del prisionero" para interpretar de forma sistemática tales problemas (por ejemplo, Hardin, 1971). Otras líneas de investigación se centraron durante aquellos años de forma más particular en contrastar empíricamente las ideas de Olson, aunque con pocas aportaciones novedosas. La obsesión sobre la plausibilidad de la hipótesis de la racionalidad para la comprensión de la acción colectiva concentraba la mayor parte de tales debates académicos, más como una confrontación entre paradigmas científicos que como una discusión sobre cómo contrastar una teoría científica (Jordana, 1992).

Así, durante algunas décadas, la falta de sensibilidad empírica en muchos desarrollos formales de la teoría de la acción colectiva, por una parte, y la resistencia a discutir "desde dentro" el paradigma de la acción racional —a veces criticado desde enfoques extremadamente empiricistas, sin alternativas teóricas concretas-, conllevó un cierto bloqueo de la teoría de la acción colectiva en las ciencias sociales. El retorno continuo de buena parte de la literatura a los conceptos fundacionales de Mancur Olson, como referencia casi indiscutible, y la ausencia de un cuerpo teórico de carácter acumulativo en este campo, son una muestra evidente de tales dificultades. No obstante, ello no impidió la aparición de algunas obras de carácter excepcional, inspiradas originalmente por las ideas de Olson, como por ejemplo The Evolution of Cooperation, de Robert Axelrod (1984), o Governing the Commons: The evolution of Institutions for Collective Action, de Elinor Ostrom (1990), por citar algunas de las más destacadas.

En los años noventa, las contribuciones a la teoría de la acción colectiva fueron más numerosas, si lo comparamos con las décadas anteriores, mostrando un resurgimiento del interés en esta teoría, aunque ello no fuera acompañado de una nueva síntesis teórica que superase claramente el marco conceptual establecido por Olson en los años sesenta. 
Algunas ideas nuevas empezaron a tomar mayor fuerza en el análisis de la acción colectiva, siendo claramente asumidas por muchos de los estudios aparecidos durante esta década. Entre estas, una cuestión clave fue considerar al dilema del prisionero como un problema concreto de la acción colectiva, sin duda muy importante, pero que no agotaba el conjunto de problemas que podría tratar la teoría de la acción colectiva. Distintas interpretaciones plantearon que otras estructuras de interacción podían capturar situaciones en las que también se producían dificultades para la aparición de acción colectiva. Así, diversos estudios examinaron las propiedades de otros juegos, considerando situaciones donde podian producirse plausiblemente problemas de participación distintos a los caracterizados por el dilema del prisionero'.

Tal vez el modelo que presentó Olson en The Logic of Collective Action (1965) contribuyó a una cierta confusión inicial, ya que éste tenía unas particularidades concretas, relativas a las características de un tipo de bienes públicos en particular, mientras que el problema que Olson presentaba, y que luego atrajo múltiples críticas y debates, parecía tener una ambición de mucha mayor generalidad. El bien público que discutía Olson en su obra suponía implícitamente distintas características: a) la necesidad de contribuciones continuas de los participantes (generando un aumento lineal de la cantidad del bien público); b) que el consumo del bien público reducía su disponibilidad (y que por tanto, generaba cierta rivalidad en el consumo), y c) que no permitía impedir el consumo de los que no contribuían a su provisión (imposibilidad de exclusión). Sin embargo, otros tipos de bienes no requieren exactamente de las condiciones planteadas por Olson, sea sobre su producción, consumo o provisión, aun manteniendo su naturaleza de bien público —si consideramos como condición mínima de un bien público la no exclusión en su consumo.

Así, en la medida en que se ha ampliado la concepción de los problemas de la acción colectiva, han surgido nuevas posibilidades de desarrollo teórico sobre las dificultades para articular acción colectiva, tanto para la producción como para el consumo de los bienes públicos. En este trabajo se revisan diversas aportaciones basadas en esta perspectiva analítica, realizadas en las últimas décadas. Un primer apartado se dedica a los intentos de clasificar conceptualmente los bienes públicos. A continuación, se discute cómo la producción de los bienes públicos influye en la acción colectiva. Un tercer apartado está dedicado al análisis de la acción colectiva desde el punto de vista del consumo de los bienes públicos. El cuarto apartado discute la interconexión entre producción y consumo examinando distintos modelos basados en la teoría de juegos. Finalmente, antes de las conclusiones, un cuarto apartado introduce diversas consideraciones sobre los límites de la hipótesis de racionalidad, y destaca el problema de la percepción para avanzar en la construcción de una teoría de la acción colectiva con mayor capacidad explicativa.

\footnotetext{
${ }^{1}$ El trabajo de Taylor y Ward (1982) posiblemente fue uno de los primeros en introducir explícitamente este punto de vista, aunque con otra perspectiva ya lo había introducido Thomas Schelling algunos años antes (1973).
} 


\section{DIVERSIDAD DE BIENES PÚBLICOS Y ACCIÓN COLECTIVA}

La identificación de los bienes públicos mediante sus características de producción y consumo constituye uno de los puntos de partida para avanzar en la teoría de la acción colectiva. En este sentido, el análisis de las propiedades de los bienes públicos se encuentra ya en los orígenes de esta teoría. Como destaca Elinor Ostrom en un trabajo reciente (2003), fue en 1954 cuando Paul Samuelson estableció una primera distinción basada en la forma de consumo del bien. Así, distinguió entre bienes de consumo privado y bienes de consumo público, según el grado de divisibilidad que presentara su consumo. Evidentemente, los bienes cuyo consumo no podía dividirse, eran considerados como bienes públicos. Además, si el consumo de un individuo no reducía el consumo de otro individuo, entraba en la categoría de bien público puro. Unos años más tarde, en 1959, Richard Musgrave apuntó un criterio distinto para analizar los bienes públicos. Se trataba del criterio de exclusión: sólo debían considerarse como tales cuando no era posible impedir a nadie el consumo del bien. Si se podía introducir algún mecanismo de exclusión sobre el consumo del bien, entonces podría generarse un mercado para regular su consumo, y por lo tanto podía ser considerado como un bien privado.

La mayor o menor facilidad para introducir la exclusión en el consumo de determinados bienes, y por tanto modificar su naturaleza de bienes públicos, constituye un elemento muy relevante para el análisis de la acción colectiva. La existencia de sofisticadas tecnologías de exclusión, o la introducción de regulaciones que introducen la exclusión (derechos de propiedad, regulación de externalidades, etc.) puede transformar radicalmente la lógica de la acción colectiva, haciendo esta completamente innecesaria, ya que el consumo puede llevarse a cabo mediante una lógica puramente individual, basada en simples percepciones individuales del interés propio (Cornes y Sandler, 1996). Cuando la exclusión es relativamente simple o poco costosa, el problema de acción colectiva puede llegar a desvanecerse. Así, quien tiene la capacidad de exclusión puede organizar la oferta del bien público de forma que se garantice su producción, aunque el consumo sea completamente conjunto. Dejando aparte los casos triviales en los que puede introducirse la exclusión de forma simple y eficaz, existen numerosos bienes cuya provisión genera problemas de acción colectiva, ya que el consumo posible (o su demanda) es superior a la cantidad de bien disponible, $\mathrm{o}$ a la que se puede producir con contribuciones voluntarias (al no ser posible excluir a los consumidores que no contribuyen).

Otra cuestión distinta es discutir como se realiza la producción del bien. Aquí encontramos una de las ideas básicas de la obra de Olson, ya que su interpretación de la acción colectiva se concentra precisamente en como se pueden producir los bienes públicos, definidos según una combinación de los criterios de Musgrave y Samuelson. Para Olson, la acción colectiva consiste en encontrar una forma exitosa de producir el bien público, a pesar de que no se pueda excluir a nadie del consumo de este bien y de que este consumo sea conjunto, sin poder dividirse. El tipo de bien público identificado por Olson implicaba que existía rivalidad en el consumo, en el sentido que el bien público se "desgastaba" por la existencia de consumidores adicionales. Sin embargo, otros 
tipos de bienes públicos, con propiedades parcialmente distintas, no encajaban bien con la interpretación de Olson sobre la lógica de la acción colectiva. Revisando este situación, Elinor Ostron señala que, después de más de 30 años de intentos sin éxito de construir una teoría que explique de forma integrada todos los problemas de acción colectiva (generados por distintos tipos de bienes), siguiendo la inspiración de Olson, tal vez sea más adecuado plantearse la necesidad de disponer de una familia de teorías estrechamente relacionadas, en lugar de seguir buscando una única teoría consistente para todos los casos (Ostrom, 2003: 242).

Una distinción clásica sobre estos aspectos es la existente entre los bienes comunales (common-pool resources) y los bienes públicos, ampliamente discutida por Elinor Ostrom (1991). La distinción principal es que el primer tipo de bienes implica un control individual sobre el consumo, mientras que el segundo tipo implica controlar la contribución, ya que el bien debe ser producido, y no sólo garantizar su flujo. En ambos casos, no es posible la exclusión para el consumo del bien a ningún individuo que lo requiera, pero la distinción radica en si el consumo de un individuo reduce el de otros, o no les afecta en absoluto (independientemente de su contribución). También encontramos un mayor grado de rivalidad en el consumo en los bienes comunales, ya que su producción viene limitada por el flujo disponible. Los comportamientos inducidos por uno y otro tipo de bien son muy distintos, y asimismo, también los resultados que podemos esperar en términos de la acción colectiva que induzca (Ostrom, 2003: 243).

Olson, por el contrario, distinguió entre bienes públicos "exclusivos" y bienes "inclusivos", refiriéndose a los bienes cuyo consumo no puede ser limitado (no-exclusivos), y sus diferencias vienen marcadas por las características que se suponen a la oferta conjunta del bien. El concepto de bien público "exclusivo" de Olson implica que éste no presenta oferta conjunta, lo que puede producir rivalidad en el consumo al aumentar el número de consumidores. Así, la intensidad de la rivalidad depende de forma dinámica de la relación entre las contribuciones para su producción y el volumen de su consumo. Para el bien "inclusivo", Olson supone que existe oferta conjunta, por lo que no aumenta la rivalidad en el consumo al aumentar el número de usuarios. En este caso, el volumen del bien puede variar según las contribuciones de los participantes y su número en la acción colectiva. Implícitamente, Olson incluye otra dimensión en su clasificación, referida a la propia naturaleza física del bien público, al considerar el posible carácter variable de su oferta conjunta (por ejemplo, un camino, un puente, generan rivalidad en el consumo sólo a partir de cierto nivel de consumo).

La clasificación de Ostrom se concentra más en la forma de producción del bien público, y menos en si este presenta una oferta conjunta. Su distinción entre bienes públicos y bienes comunales tiene que ver con la forma de producción: Ostrom considera que los bienes comunales tienen interdependencia en el consumo (el consumo de uno puede reducir el consumo de otro), ya que la producción tiende a ser fija, mientras que esta interdependencia no existe en el caso de los bienes públicos en los que la producción depende de las contribuciones (el consumo de uno no tiene porque reducir el consumo de otros). En la clasificación de Ostrom también encontramos una distinción basada en 
el grado de rivalidad, de forma similar a Olson, aunque en este caso la rivalidad es consecuencia del consumo del bien (dada la idea de sustracción del bien comunal), mientras que para Olson la rivalidad aparece en la medida que las contribuciones a su producción y el grado de consumo no coinciden.

Una forma de aproximarnos mejor a todo este entramado teórico relativo a la acción colectiva y los bienes públicos puede ser utilizar una definición de bien público más general de lo que a menudo ha sido utilizado en la literatura sobre la teoría de la acción colectiva. Por ejemplo, siguiendo la propuesta de Holzinger (2003: 175), podemos considerar como bienes públicos todos aquellos bienes caracterizados por la presencia de externalidades significativas; en definitiva, todos aquellos bienes que no son de naturaleza estrictamente privada. Luego, encontramos bienes que con propiedades distintas, desde los bienes públicos puros, con no-exclusión absoluta y sin rivalidad, hasta los bienes de club a los que es posible aplicar la exclusión en su consumo. La figura 1 nos muestra esta diversidad de bienes públicos, ubicados según los dos criterios tradicionales, su grado de no-exclusión y de oferta conjunta, y un criterio adicional, relativo al flujo de producción del bien.

De la discusión en torno a las clasificaciones de bienes públicos podemos intentar extraer algunas enseñanzas. Primero, que posiblemente no exista una clasificación mejor

Figura 1.

Relación entre producción, exclusión y rivalidad

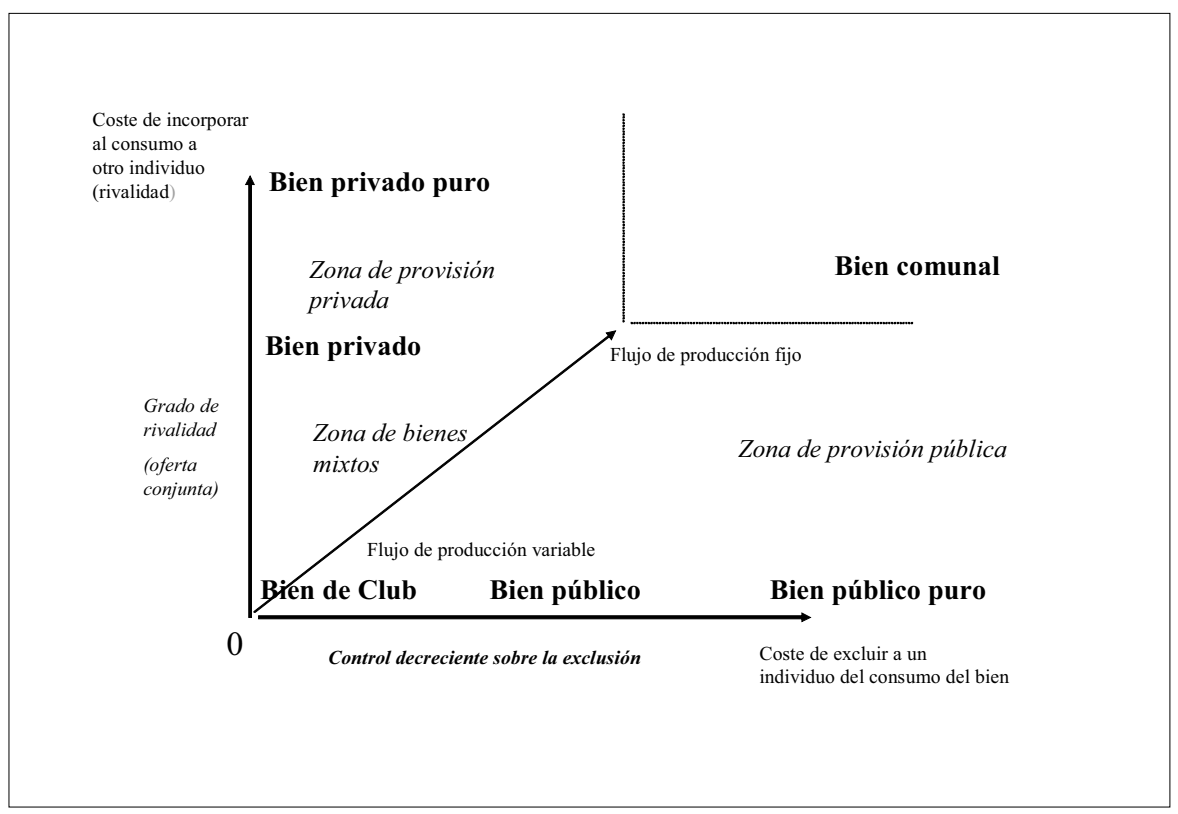


que otra, sino que ambas son particularmente adecuadas para identificar problemas específicos para la acción colectiva (la interdependencia en el consumo en el caso de Ostrom, el ajuste entre producción y consumo en el caso de Olson); en segundo lugar, en un proceso de acción colectiva para obtener bienes públicos observamos más dimensiones de las que permite apuntar una simple distinción dicotómica, y seguramente la distinción en tres dimensiones, presentada en la figura 1, ayude a clarificar los dilemas existentes. Finalmente, que las clasificaciones sobre bienes públicos son útiles, sin duda, para interpretar la acción colectiva, ya que nos apuntan claramente una idea central: la importancia que tienen las características de los bienes públicos para explicar los problemas de la acción colectiva.

\section{LAS FUNCIONES DE PRODUCCIÓN DE BIENES PÚBLICOS}

La identificación de las propiedades del bien público es una cuestión muy relevante para el análisis de la acción colectiva, dado que las hipótesis sobre el comportamiento individual pueden conectarse directamente con estas propiedades, para identificar los problemas particulares de la acción colectiva. Una de las formas de conexión consiste en la propia producción del bien público. En la medida que así sea, la adecuada definición de la función de producción permite identificar qué problemas genera cada tipo de bien público para impulsar una acción colectiva. La función de producción para producir un bien público (o un bien comunal) puede tomar formas muy diversas, pudiendo ser incorporadas distintas variables relativas al proceso de producción del bien y las contribuciones de los participantes individuales en la función. Una simple función de producción lineal, donde la oferta conjunta es total, implica que la suma de todas las contribuciones individuales a la producción del bien público deben multiplicarse por un ratio de transformación, siendo su resultado el volumen de bien público producido. Con la no-excusión, el resultado obtenido podrá ser aprovechado por todo el mundo, sean o no contribuyentes. Si este ratio es superior a la unidad, podemos suponer que la contribución de un determinado porcentaje de participantes, será suficiente para igualar el beneficio o consumo individual de todos los individuos interesados en el bien público. Por debajo de este porcentaje, los que efectivamente contribuyen no compensaran totalmente su esfuerzo con los beneficios individuales que consigan con el consumo del bien público; por encima del porcentaje, la compensación será positiva.

Evidentemente, si el grado de oferta conjunta del bien público no fuera total, existiendo un cierto desgaste en el consumo del bien, o una cierta rivalidad entre los consumidores, la relación propuesta podría complicarse algo más, ya que deberíamos considerar también el impacto del consumo sobre la oferta de bien público. Por ejemplo, si suponemos que hay un cierto desgaste del bien a medida que aumenta el número de consumidores, deberíamos sustraer a la cantidad total del bien público disponible, al desgaste generado por el número de consumidores (siendo este mayor o igual que el número de contribuidores). 
Estas relaciones pueden hacerse cada vez más complicadas, siendo ya necesario abordarlas con un tratamiento formal para su análisis. Por ejemplo, cuando el desgaste del bien público — debido al consumo— no tiene un carácter lineal y se acelera a medida que crece el número de consumidores o el consumo de estos; o cuando se incluyen otras características del bien y la función toma formas más complejas. Tenemos el caso de los bienes escalares (por paquetes), que necesitan un volumen mínimo de contribuciones para que puedan ser consumidos (por ejemplo, la construcción de un puente, o la formación de una cadena humana uniendo dos puntos), y una vez creados no les afecta el aumento de su consumo (tal vez hasta el momento en que se produzca un colapso, si aumentan mucho los usuarios). En tales casos, alcanzar una proporción mínima de contribuyentes es algo realmente crítico, ya que si no se alcanza, los que contribuyen no sólo tendrán pérdidas netas, sino que no obtendrán en absoluto el bien público que se proponen disfrutar.

Como vemos, es importante distinguir entre el consumo y la producción de los bienes públicos. Si con el primero observamos el grado de oferta conjunta del bien público, con el segundo consideramos las características del proceso de producción del bien. La tecnología de agregación —nos referimos a la forma como las contribuciones individuales hacen posible la producción de un determinado bien público—, tiene también un impacto relevante en la interacción entre los individuos participantes, y según sus propiedades, puede producirse un problema de acción colectiva, cosa que ha sido destacada ya por algunos autores (Taylor, 1987; Holzinger, 2001). Nuestro conocimiento sobre la producción del bien público puede facilitar el análisis de la acción colectiva, ya que cambios en la tecnología de producción pueden transformar la naturaleza de los problemas de acción colectiva.

Marwell y Oliver (1993), en un conocido trabajo sobre acción colectiva y masa crítica, desarrollan numerosos argumentos insistiendo en que no existe un solo problema de acción colectiva, explicable mediante un modelo analítico concreto. Consideran que es necesario distinguir conceptualmente distintos tipos de acción colectiva, e identificar los factores relevantes en cada uno de ellos. De forma explícita, señalan que "Nuestro punto de partida es que no hay principios generales de acción colectiva: deben tomarse algunos parámetros de tipos de acciones particulares, y luego se pueden examinar los efectos de otros factores como el tamaño del grupo" (Oliver y Marwell, 2001: 296). Su punto de vista es que las contribuciones producen un determinado volumen de bien público, generalmente de forma no lineal. Suponen que existe una relación entre el volumen y el número de las contribuciones, y que la contribución de cada participante puede ser variable. Así, para producir el bien público se necesita combinar participantes y contribuciones en proporciones específicas. Al mismo tiempo, calculan el beneficio (aprovechamiento del bien público) para el contribuidor, sin identificar necesariamente como se produce éste: sea directamente por la producción (considerando oferta conjunta plena), sea por una determinada combinación de la forma de producción y efecto de consumo (cuando no hay oferta conjunta completa), que la función implícitamente establece para el nivel de análisis individual. Su preocupación general es el análisis de la interdependencia en relación con 
las funciones de producción (2001: 295), y en este sentido, la mayor parte de su obra se centra en analizar los procesos de decisión secuencial, cuando los jugadores disponen de información sobre las elecciones realizadas previamente por otros actores.

Hemos visto ya la importancia de las funciones de producción de bienes públicos para identificar los problemas de la acción colectiva. Por este motivo, vamos a profundizar algo más en las características de estas funciones, que pueden ser extremadamente variadas. Intentando sistematizar más la cuestión, debemos averiguar en primer lugar si las contribuciones al bien público por parte de cada individuo son aditivas (sí con más contribuciones aumenta la cantidad producida, y de qué forma aumenta); en segundo lugar, analizar la forma específica de la función de producción, y finalmente, identificar si las contribuciones son homogéneas o heterogéneas, en el sentido de que cualquier aportación puede ser valorada igual (p.e., dinero), o tiene un valor distinto (p.e., habilidades personales).

Hirshleifer (1983), en una de las primeras contribuciones a la discusión sobre las funciones de producción de bienes públicos, ya señalaba que pueden existir casos donde no funcione la propiedad aditiva. Casos en los que, por ejemplo, el bien sólo se produzca con una única contribución individual, de un valor mínimo determinado (un voluntario que consiga matar a un dragón, por ejemplo). Otra posibilidad es que el bien se produzca al nivel que determine la contribución menor, o la peor, aunque sean también necesarias otras contribuciones (al transmitir un mensaje a través de intermediarios, su fiabilidad depende del vínculo más débil). $\mathrm{O}$ al revés, que el nivel de provisión del bien venga determinado por la mejor contribución (propuestas para conseguir el mejor título de una obra colectiva). Todos estos son ejemplos extremos, pero nos dan a entender la variabilidad en la producción del bien público. Los bienes públicos de carácter escalar también muestran la característica de no ser aditivos, por lo menos de forma completa. En este sentido, pueden sumarse las contribuciones hasta llegar al nivel necesario para la aparición del bien público, pero luego contribuciones adicionales no tiene ningún efecto positivo sobre la disponibilidad del bien (a no ser que supongamos que el bien se produce por tramos 0 fases y deban acumularse contribuciones suficientes para alcanzar el siguiente tramo).

Como una forma de definir específicamente las funciones de producción, Marwell y Oliver (1993) presentan en su libro una serie de funciones de producción no lineares, a las que llaman "acelerantes" y "desacelerantes" inspiradas en los modelos de elecciones binarias de Shelling (1973), donde relacionan los costes de la contribución con los beneficios esperados para los contribuidores. En su trabajo, muestran cómo las funciones de producción toman diversas formas: cóncavas, convexas, cuadráticas, o incluso formatos más generales como ecuaciones de tercer orden. Por ejemplo, en las funciones cóncavas (desacelerantes), cada contribución adicional reduce el beneficio por individuo a partir de un cierto volumen -lo que facilita el impulso inicial para la producción del bien público, aunque luego surjan problemas para sostener tal producción. En las funciones convexas, es al revés -siempre considerando que cada contribuidor adicional es también un consumidor adicional del bien público. Así, el típico ejemplo del éxito de una huelga como acción colectiva tiene que ver con una función convexa. Cuanto mayor sea la proporción 
de participantes sobre el total de trabajadores, aumenta más que proporcionalmente la probabilidad de éxito de la huelga, y finalmente, también la probabilidad de obtener las reivindicaciones - entendidas como un bien público.

Cuando suponemos el carácter heterogéneo de las contribuciones, debemos considerar los casos de aportaciones de recursos no intercambiables en la producción del bien colectivo. Sin embargo, esta perspectiva complica mucho los modelos y dificulta enormemente el trabajo con esquemas deductivos. La realización de simulaciones constituye entonces una vía mucho más atractiva para identificar en qué condiciones o contextos se pueden resolver problemas de acción colectiva. La heterogeneidad, sin embargo, puede explicar con facilidad situaciones de acción colectiva en las que bienes con una fuerte oferta conjunta, son producidos por pocos contribuyentes en un grupo muy grande. Oliver y Marwell $(1993,2001)$ desarrollan diversos trabajos con simulaciones sobre el papel de la heterogeneidad de los recursos de participantes en la acción colectiva, junto con otras variables (costes de organización, densidad y centralización de las redes, heterogeneidad de intereses), con el fin de observar su influencia en las probabilidades de creación de acción colectiva. Los resultados de la heterogeneidad también han sido explorados por Heckathorn (2002), quien analiza en qué medida las diferencias fragmentan la producción de bienes públicos, mostrando cómo pueden generar incluso antagonismos.

Para finalizar este apartado, cabe destacar una aproximación distinta a la producción, mostrada por Esteban y Ray (2001) al desarrollar un modelo formal, desde una lógica muy centrada en el comportamiento de los grupos de intereses, donde analizan, en términos de coste marginal, las variaciones en la contribución individual. Su idea central es que la contribución individual no es lineal, sino que configura una función convexa, en la que el esfuerzo de una contribución adicional de recursos propios a la producción del bien es cada vez más costosa, sea en tiempo o en dinero. El modelo que presentan permite identificar el grado de convexidad - la elasticidad del coste marginal- de las contribuciones individuales para la producción del bien (cero sería una relación puramente lineal). La forma en que conciben la oferta del bien público se centra identificar el esfuerzo de los individuos como contribuyentes, y su relación entre contribución y consumo del bien público (teniendo en cuenta el grado de rivalidad de este).

Al relacionar el grado de rivalidad de un bien público con el coste marginal de la contribución, Esteban y Ray (2001) identifican un espacio de posibilidades en el que no se cumple la clásica predicción de Olson sobre la reducción en la provisión del bien público al aumentar el tamaño del grupo, y por el contrario, demuestran que un incremento en el tamaño del grupo aumenta la efectividad del grupo (en términos de aumento de la probabilidad de obtener el bien público o de una determinada cantidad de éste). En otras palabras, para los bienes con escasa o nula rivalidad y costes marginales de contribución crecientes, un aumento en el tamaño del grupo implicará mayor provisión del bien público. Esteban y Ray muestran que la tesis de Olson relativa a que los grupos pequeños son más efectivos para la provisión de bienes públicos no se puede generalizar, ya que depende de las características del bien — básicamente, su grado de rivalidad - y del esfuerzo de los individuos para la producción del bien público (o la utilidad marginal de su consumo). 
Sólo en determinadas circunstancias se cumple la tesis de Olson —alta rivalidad del bien y coste marginal bajo-, y aún depende del tamaño inicial del grupo. Como señalan los autores del trabajo, la tesis de Olson se sostiene sólo de forma general en la medida en que se suponga la linealidad de la función de costes, pero no cuando suponemos que un esfuerzo adicional es más costoso para un individuo que el esfuerzo inicial (2001: 671).

En todo caso, su modelo aborda el análisis formal de forma continua y no dicotómica, lo que les permite discutir con mucha mayor precisión las interacciones y los equilibrios entre las distintas variables clave que identifican. Si embargo, no distinguen entre contribución y producción del bien, e implícitamente suponen que las contribuciones individuales -con un coste marginal creciente - son puramente aditivas, lo que conduce a un aumento de su "volumen" al aumentar el tamaño del grupo. El modelo no entra en identificar la tecnología del bien público, y en especial su función de producción específica, pero su análisis no lineal de la contribuciones individuales abre unas perspectivas sugerentes para complementar las interpretaciones basadas en la tecnología de la producción ${ }^{2}$.

\section{RIVALIDAD Y CONSUMO EN LA ACCIÓN COLECTIVA}

Antes de analizar las relaciones de interdependencia entre el consumo y la producción del bien público, exploramos de forma separada los problemas relativos al consumo, ya que nos permite identificar algunos elementos básicos que en ocasiones pueden pasar desapercibidos. Así, en este apartado nos centramos en el análisis de la acción colectiva desde la perspectiva de cómo se asigna el consumo del bien público, un problema que surge cuando su oferta conjunta no es completa, y que ha sido poco explorado por la literatura especializada. El grado de desgaste del bien público influye, claro está, en cómo se reparte su consumo. No hay que olvidar que estamos considerando bienes públicos, que no son fragmentables de forma individual, pero que pueden mostrar algún grado de rivalidad en su oferta conjunta. La rivalidad en el consumo del bien público puede generar incentivos para determinados comportamientos, dependiendo de las características del propio bien y sus patrones de consumo. Habrá numerosos casos triviales, pero otros pueden mostrar problemas relevantes, tanto sobre la coordinación entre los consumidores, como sobre sus posibles conflictos. A ello debemos añadir que para analizar el

\footnotetext{
2 Encontramos una muestra de ello en una extensión del modelo de Esteban y Ray, desarrollada por Pecorino y Temini (2004). Consideran la existencia de unos costes fijos de participación que deben ser aportados por los contribuidores potenciales antes de que sus contribuciones tengan un impacto en la provisión del bien público. Su resultado es que la existencia de costes fijos reduce de forma significativa la tendencia a que los grupos grandes produzcan más cantidad (o aumente la probabilidad) de bienes públicos, y sólo en los casos en que no existe rivalidad se mantiene la tesis de Esteban y Ray. Cuando existe un nivel importante de rivalidad, encuentran que se mantiene la tesis tradicional de Olson sobre la tendencia decreciente de la provisión del bien público al aumentar el tamaño del grupo, independientemente del nivel de convexidad de la función de contribución (2004: 19-23).
} 
consumo del bien público podemos considerar también la utilidad individual, relativa a como el consumidor satisface su consumo del bien. En la medida que su consumo vaya aumentando, posiblemente decrezca su utilidad, lo que supone un límite en la práctica, independientemente de cómo sea su participación.

En este sentido, Ostrom (2003: 247) plantea la importancia de las funciones de reparto del bien público para entender los problemas de la acción colectiva, señalando que el reparto en el consumo puede ser proporcional a la participación, o puede derivarse de consideraciones externas (como la ubicación espacial o temporal del consumidor), o también de la existencia de reglas institucionales que asignen la forma específica de consumo. Precisamente las reglas que asignan el consumo de bienes públicos pueden constituir una fórmula para solucionar algunos de los problemas mencionados, aunque para ello es preferible conocer antes la naturaleza del bien. En todo caso, para examinar todas estas variables, es mejor suponer constante la producción del bien público, sin valorar cómo pueden influir las variaciones en la producción sobre el consumo del bien público.

Para nuestra discusión en este apartado, podemos suponer que el consumo del bien público por parte de los individuos interesados no es lineal en todos los casos. En muchas ocasiones, al aumentar el consumo del bien público, la utilidad para el consumidor aumenta de forma decreciente. En otras palabras, podemos suponer que la utilidad marginal del consumo es la que justifica el grado de convexidad de la función de contribuciones individuales. Esta dinámica puede mostrar una forma convexa, y tener un impacto relevante en las funciones de reparto antes mencionadas, y también puede establecerse una relación con el grado de rivalidad del bien.

En su modelo, Esteban y Ray (2001) proponen una fórmula para identificar el grado de oferta conjunta del bien público, tomando como referencia la relación entre la porción de un bien que no muestra rivalidad en el consumo (y por tanto, a la que no afecta el tamaño del grupo) y la porción que muestra rivalidad en el consumo (que depende del tamaño del grupo). Luego, si relacionamos la elasticidad de la utilidad marginal del consumo con una medida del grado de rivalidad de un bien público, podemos considerar que si el consumo de un bien por parte de un individuo tiene escasa elasticidad (un mayor consumo reduce rápidamente su utilidad) y el grado de rivalidad no es muy intenso, las funciones de reparto tenderán a descontar tales condiciones, haciendo más fácil el establecimiento de reglas para la asignación del consumo entre los participantes interesados. En otras palabras, para los bienes con escasa o nula rivalidad y utilidad marginal decreciente en el consumo, encontraremos una menor posibilidad de conflicto que en el caso de bienes con mayor rivalidad, o con utilidad no tan decreciente en su consumo.

La asignación de recursos hídricos es uno de los ejemplos más utilizados por $\mathrm{E}$. Ostrom en sus estudios sobre las funciones de reparto (p.e., Ostrom, Gardner y Walker, 1997), donde muestra que las reglas institucionales deben ser compatibles con el contexto material del consumo (cercanía al recurso, grado de necesidad, etc.), para superar las dificultades de distribución de agua en los sistemas de irrigación donde la capacidad acceso al recurso es muy distinta entre los diferentes implicados. A todo ello, deberíamos 
añadir también la necesidad de considerar la función de utilidad individual en el consumo del bien -ya que puede reflejar también distintos elementos culturales y morales que activen comportamientos distintos a similares condicionamientos externos- favoreciendo una cierta auto-limitación del consumo, incluso entre los usuarios con el acceso más privilegiado al recurso. En todo caso, cabe añadir que una de las conclusiones básicas de Ostrom, en relación al funcionamiento de las reglas de reparto, es la importancia de la existencia de mecanismos de participación de los propios consumidores en el gobierno de los sistemas de reparto, especialmente por su mayor flexibilidad para ajustarse a todo tipo de cambios.

\section{BIENES PÚBLICOS Y MODELOS DE INTERACCIÓN}

En las secciones previas hemos estado discutiendo las características de las funciones de producción y de consumo de los bienes públicos, sin entrar en la interdependencia entre las dos partes, la producción y el consumo de los bienes públicos. En este sentido, podemos entender las estructuras de interacción desarrolladas por la teoría de juegos como una forma de representar estas interdependencias entre producción y consumo de los bienes públicos. La teoría de juegos permite integrar analíticamente los componentes individuales que incluyen algún supuesto de racionalidad, analizando los posibles equilibrios en la interacción entre individuos. No se trata tanto de crear 0 aplicar directamente modelos sobre los problemas de la cooperación y la coordinación entre individuos, sino de algo más concreto; de desarrollar unos modelos interpretativos que incluyan, de forma explícita, los elementos más significativos de la producción y el consumo de distintos tipos de bienes públicos de forma interdependiente, suponiendo, claro está, la existencia de una racionalidad básica por parte de los actores que toman decisiones de forma simultanea sobre cómo contribuir a la producción y consumir el bien público.

Aunque la mayor parte de la literatura ha tendido a identificar el juego del dilema del prisionero con el problema básico de la acción colectiva, al mostrar la tensión entre la racionalidad individual y la racionalidad colectiva; la variedad de problemas de acción colectiva que puede generar la provisión de bienes públicos no se termina con este juego. Sin lugar a dudas, tal simplificación ha sido muy útil para explorar y discutir la interdependencia entre contribución y consumo de bien publico en situaciones mixtas de de conflicto y cooperación. La exploración de otros modelos fue tratada por diversos autores ya desde los años setenta, identificando juegos distintos al dilema del prisionero y planteando que determinadas características de los bienes públicos podían producir distintos tipos de estructuras de interdependencia para asegurar su provisión (Sandler, 1992; Snidal, 1985; Taylor y Ward, 1982; Taylor, 1987). En general, estos trabajos tomaban como punto de partida la identificación de distintos bienes públicos, especificando las variaciones en las propiedades de no-exclusión y oferta conjunta.

Un ejemplo de estas distinciones es la clasificación establecida por Ostrom, ya mencionada, que distingue entre bienes públicos y bienes comunales, y sugiere que 
cada tipo de bien conlleva un modelo de interacción distinto para coordinar las acciones de los individuos que desean obtener el bien (Ostrom, 2005; Ostrom et al., 1994). Las interacciones generadas por los bienes comunales han sido exploradas también por otros autores, a menudo insistiendo en que el modelo del dilema del prisionero no caracterizaba el problema de acción colectiva generado por estos bienes (Hees, 2000). Diversos trabajos intentaron identificar qué tipos de juegos podían representar mejor la situación generada en situaciones de desgaste del bien público, como los juegos de coordinación (Runge, 1984), o el dilema del voluntario (Rapoport, 1988).

Sin embargo, más allá de estos intentos de vincular un juego a un tipo de bien público con características particulares, una clasificación más compleja, inspirada en un trabajo de Taylor y Ward (1982), nos sirve para ilustrar nuestro argumento sobre la variedad de problemas de acción colectiva y la teoría de juegos, y nos introduce también en una nueva perspectiva sobre cómo vincular variedades de bienes públicos con el análisis de la acción colectiva mediante el empleo de modelos simples de teoría de juegos. La clasificación se basa en una característica de la producción del bien -si es continua 0 escalar (se produce a medida que se consume, o sólo en un momento dado) - y en una característica de su consumo, el grado de rivalidad o de oferta conjunta del bien público. Con estas dos distinciones, se pueden identificar cuatro tipos de juegos distintos, cada uno reflejando distintos problemas de acción colectiva. Así, se identifica el juego del gallina con situaciones de provisión continua y baja rivalidad del bien público; el juego del héroe con la provisión escalar y baja rivalidad; el juego del prisionero con la existencia de alta rivalidad y provisión continua en relación al bien público; y finalmente, el juego de la seguridad con la provisión escalar y la presencia de alta rivalidad en el consumo del bien.

Implícitamente, en este esquema suponemos que cada forma de producción induce distintas percepciones para los participantes en una acción colectiva: en los casos de producción continua pueden darse situaciones de disponibilidad de bien público por debajo de lo deseado. En el caso los bienes escalares, no existe esta percepción: o se consigue el bien o no se consigue, pero no hay situaciones intermedias. Con relación al consumo, encontramos una situación análoga: si el bien público no se "desgasta" con el consumo, o no hay rivalidad entre los consumidores (suponiendo que no puede excluirse a nadie), la actitud de los individuos para participar en su provisión también puede ser distinta. Cuando hay rivalidad, la exigencia de cooperación activa para mantener el bien público es muy necesaria, cuando no hay rivalidad, esta exigencia sin duda es menor, y con algún mecanismo de coordinación entre los jugadores se puede asegurar la existencia del bien público. En otras palabras, la interdependencia para asegurar el bien público es más intensa en los casos en los que hay desgaste del bien como fruto del propio consumo (y por tanto, rivalidad directa o indirecta).

La relación entre funciones de producción y la teoría de juegos ha sido explorada por diversos autores, ya que constituye una cuestión clave para identificar la interdependencia entre intereses individuales y resultados colectivos. Mediante la definición de los pagos de los juegos (payoff), podemos incorporar elementos de las funciones de producción del bien público, de igual modo que en la definición del proceso de calculo individual también 
podemos incorporar percepciones sobre el riesgo, sobre los costes marginales de la contribución o sobre la utilidad del consumo del bien público. El juego del prisionero ha sido muy utilizado en la literatura, a través diversas modalidades y formulaciones, para analizar los problemas de la acción colectiva, y es en las funciones de pagos que especifican los resultados - en términos de beneficios de bien público — para las distintas estrategias de los jugadores (entendidas como su grado de contribución a la producción del bien público) donde se introducen también hipótesis sobre la naturaleza del bien público y su forma de producción. Por ejemplo, un esfuerzo pionero en este sentido, cuando los juegos incluyen jugadores y sólo dos opciones de contribución (cooperar y no cooperar), lo encontramos con los conocidos diagramas de Scheling $(1973)^{3}$.

Una forma simple y sugerente de aplicar estos planteamientos fue propuesta por Aggarwal y Dupont en su artículo "Goods, Games and Institutions" (1999) al plantear estructuras de juegos que correspondían a diferentes supuestos sobre los costes y beneficios de los bienes públicos. Estos autores tomaron la distinción de Ostrom (1994) entre bienes públicos y bienes comunales, e identificaron por el lado del consumo la variable de desgaste del bien público, mientras que por el lado de la producción identificaron su carácter incremental (o escalar). Con estos criterios, construyeron un esquema simple, con dos jugadores y dos opciones para cada uno: contribuir o no contribuir a la producción de bien público. Los resultados (payoff) derivados de la contribución de ambos actores implican que el bien público se produce. Mientras, si uno contribuye y el otro no, el resultado dependerá del tipo de bien público, y si ninguno de los dos contribuye, evidentemente no se producirá el bien público. Por otra parte, consideran que el comportamiento de los jugadores depende de los recursos de que disponen, de los costes específicos de la contribución y de los beneficios que se obtengan del bien público, teniendo en cuenta que los beneficios no sólo alcanzan a los que contribuyen, sino a todos los participantes (1999: 396-7).

A partir de este esquema analítico, Agrawall y Dupont identificaron una serie de juegos, según las variaciones en los costes, beneficios y recursos de los participantes en la acción colectiva (no pueden perder más de lo que disponen, en el caso de que contribuyan sin llegar a producir el bien público). Así, por ejemplo, muestran que a medida que van aumentado los beneficios generados por el bien público, el juego se transforma: de un juego sin salida (Deadlock), se pasa al juego de la seguridad. También complican los juegos posibles considerando la provisión de recursos de los actores. Con ello surgen situaciones donde los jugadores perciben estructuras de interacción distintas. Por ejemplo, para un jugador sus posibilidades de acción configuran un juego del gallina, mientras que

\footnotetext{
${ }^{3}$ Un intento de formulación más general del modelo de Schelling es la propuesta de definir las funciones de pagos en forma de ecuaciones cúbicas (Szilagyi, 2000). Donde las funciones cóncavas, convexas o con formas más complejas (U-shaped) son casos particulares, según los valores que tomen los distintos comportamientos posibles de los jugadores (cooperan todos, cooperador aislado, no coopera nadie, no cooperador aislado).
} 
el otro, percibe un dilema del prisionero. Dada la distribución de pagos del juego, podemos identificar en cualquier caso un equilibrio de Nash. Finalmente, los autores presentan una taxonomía en la que identifican diversos juegos según la importancia de los beneficios, por una parte, y la relación entre el coste de la contribución y el nivel de los recursos de los individuos que participan en la acción colectiva, por otra (si los recursos son mayores o menores que el coste de la contribución, tomando por separado cada actor). En esta taxonomía muestran, por ejemplo, que el juego del dilema del prisionero sólo representa adecuadamente un tipo particular de interacción para producir el bien público: cuando los beneficios son medianos (debido a la existencia de rivalidad en el consumo), y los recursos disponibles son mayores que el coste de la contribución (por lo menos para uno de los actores). Otros juegos identifican el problema para la provisión del bien público cuando se dan circunstancias distintas en relación a los costes, beneficios y los recursos de los actores implicados (Agrawal y Dupont, 1999: 401).

Con su propuesta analítica, estos autores muestran que la relación entre la producción del bien y el consumo de éste puede generar estructuras de interacción diferentes. Según la importancia de los beneficios y del coste relativo de la contribución, el comportamiento estratégico de los actores no será el mismo, y sus preferencias variarán, reflejando la existencia de una serie de percepciones sobre la interdependencia entre contribución individual y beneficios del bien público, que al mismo tiempo toman en cuenta el comportamiento estratégico de los otros actores.

Otro trabajo que desarrolla un planteamiento similar es el artículo de Holzinger "Common Goods, Matrix Games and Institucional Response" (2003). La autora considera también que distintas características de los bienes públicos, sus costes y beneficios, conducen a diferentes problemas de acción colectiva, y señala específicamente que las variaciones en los contextos sociales de los actores que producen los bienes tienen también un impacto en los problemas de la acción colectiva. aHolzinger critica el trabajo de Aggarwal y Dupont señalando que algunos factores de su esquema teórico no están claramente especificados, en particular su propuesta relativa a la transición desde la identificación de los bienes públicos a la definición de los juegos que proponen en su artículo, y la función de transformación de las contribuciones individuales a la formación de los bienes públicos. Por ello, considera que su clasificación propuesta de juegos no es válida.

Una de sus críticas consiste precisamente en señalar que no es suficiente con identificar los costes de la participación, sino que es necesario identificar con claridad la función de producción del bien -como ya hemos examinado previamente. Por otra parte, también destaca distintos problemas de especificación de las variables en los juegos propuestos por Aggarwall y Dupont, en particular la variable relativa a la disponibilidad de recursos individuales por parte de cada jugador (2003:180-2). En este sentido, Holzinger destaca que la estructura de los juegos viene determinada realmente por la relación entre costes y beneficios individuales, con respecto a los costes y beneficios colectivos, considerando fijas otras variables que puede influir en la interacción (simetría de los jugadores, linealidad de las contribuciones en la producción de bien público, etc.). Así, por ejemplo, señala que con estos supuestos, encontraremos una estructura de interacción con el patrón del dilema 
del prisionero cuando los costes totales sean mayores que los beneficios individuales, y los beneficios netos sean positivos; cuando los costes globales sean inferiores a los beneficios individuales, por el contrario, señala que estructura de interacción será la del juego del gallina. La polémica entre ambos autores continuó en sendas notas a los editores de la revista European Journal of Internacional Relations, donde se había publicado el artículo de Holzinger unos meses antes. Los puntos de discrepancia se hicieron incluso más visibles entonces: por una parte la cuestión de la divisibilidad de los bienes públicos (si se produce en una sola unidad, por bloques, o en cantidades variables), y por otra con relación a cómo analizar el papel de los recursos de que disponen los jugadores 4 .

Esta discusión sobre cómo analizar la relación entre bienes públicos y estructuras de interacción representadas por juegos de estrategia, es sin duda muy útil para ilustrar algunos de los problemas centrales que afronta el análisis de las interdependencias entre producción y consumo en la acción colectiva. En particular, la discusión sobre divisibilidad o no de los bienes públicos, o la no divisibilidad para su impulso inicial, representa una diferencia significativa para los modelos de ambos autores. Esta diferencia constituye un supuesto básico para estructurar los juegos que se proponen (para bienes públicos continuos, pueden producirse cantidades iniciales con contribuciones individuales pequeñas; para bienes públicos discontinuos, las cantidades iniciales deben ser fijas, y ello limita en alguna medida los posibles juegos a considerar). Sin duda, podemos considerar ambas aportaciones valiosas para ser aplicadas a tipos de bienes públicos distintos, pero hay que tener presente que también muestran un grado de solapamiento importante entre ellas.

Posiblemente una de las propuestas más sugerentes y sistemáticas para relacionar la producción de bienes públicos con su consumo la encontremos en el trabajo de Douglas Heckathorn "The Dynamics and Dilemas of Collective Action" (1996), en el que utiliza la teoría de juegos para conectar con una única estructura ambas dimensiones. Su objetivo es mostrar cómo las funciones que representan los pagos o beneficios a los participantes, definen también distintos espacios de interacción y estos pueden ser identificados con distintos tipos de juegos. Heckathorn propone una sistematización —que sugiere exhaustiva- de los problemas de la acción colectiva, identificando cinco juegos distintos, entre los que se incluye el dilema del prisionero, y señala dos vías distintas para solucionar tales problemas. Por una parte, intentando resolver el dilema dentro del propio juego, y por otra

\footnotetext{
${ }^{4}$ En relación con el primer punto, mientras Agrawal y Dupont (2003: 476) sostienen la importancia de considerar la no divisibilidad de muchos bienes públicos, como un problema central para la acción colectiva, Holzinger (2003: 479-80) insiste en que, aunque existan casos de indivisibilidad en la provisión de bienes públicos, esta no puede ser una distinción general para analizar los problemas de la acción colectiva. Sobre el segundo punto, Holzinger (2003: 480-81) sostiene que en el modelo de Agrawal y Dupont existe información incompleta sobre los recursos que disponen cada jugador, y ello conduce a que los individuos no puedan calcular bien los equilibrios posibles, tal como está planteado el juego, ya que no saben si el otro jugador dispone de recursos suficientes para contribuir; mientras que Agrawall y Dupont (2003: 476-7) apuntan que si ambos actores contribuyen a producir un bien público, se produce un sobregasto, y estos pueden recibir de vuelta la cantidad no utilizada.
} 
parte, modificando el juego, y con ello, facilitando la resolución del problema planteado. La propuesta de Heckathron está influida sin duda por los trabajos de Marwell y Oliver (1993) y su identificación de distintas funciones de producción, aunque toma una dirección distinta. Para Heckathorn (1996: 254-7), el nivel de producción de un bien público depende de la proporción de individuos que contribuyen, que se relaciona con el volumen de bien público producido mediante una función monótona creciente. Esta función puede tomar valores distintos, según la tecnología de transformación, y se expresa en un exponente (F) en la relación propuesta. Así, el nivel de bien público producido sería:

$$
L=1-(D / N)^{F}
$$

Donde $\mathrm{D}$ es el porcentaje de no contribuidores y $\mathrm{N}$ el total de participantes del grupo que consumen el bien público. Cuando $\mathrm{F}>1$, la función es desacelerante (a más contribuidores, menor proporción de la cantidad de bien producido); y cuando $\mathrm{F}<1$, la función es acelerante: a más contribuidores, más que proporcional será la producción del bien público. Este modelo asume algún supuesto simplificador, sin duda, como la homogeneidad de las contribuciones, la forma de la función (que, por ejemplo, no permite pensar en reducciones del bien público al aumentar el número de contribuyentes); y también supone una contribución fija para cada individuo. Sin embargo, el modelo identifica claramente un amplio conjunto de posibilidades para la producción del bien público. Heckathorn transforma esta función en un sistema de juegos de $2 \times 2$, al considerar la existencia de dos participantes, y tres posibles resultados: ambos contribuyen (producción completa del bien), ninguno contribuye (no producción del bien), y un solo contribuyente (producción del bien en la proporción $\left.1-(1 / 2)^{\mathrm{F}}\right)$. Con este marco simplificador, propone una matriz de pagos para ambos jugadores, siguiendo el esquema convencional en el que a los beneficios de cada jugador por disfrutar del bien público (V), debemos descontar el coste de su contribución (K):

Tabla 1.

Matriz de pagos modelo Heckathron

\begin{tabular}{|l|c|c|}
\hline & Sí contribuye & No contribuye \\
\hline Sí contribuye & $\mathrm{R}=\mathrm{V}-\mathrm{K}$ & $\mathrm{S}=\mathrm{V}\left(1-(1 / 2)^{\mathrm{F}}\right)-\mathrm{K}$ \\
\hline No contribuye & $\mathrm{T}=\mathrm{V}\left(1-(1 / 2)^{\mathrm{F}}\right)$ & $\mathrm{P}=0$ \\
\hline
\end{tabular}

Fuente: Heckathorn (1996: 256). 
Partiendo de esta matriz de pagos, Heckathorn proponen unas nuevas funciones que relacionan el exponente de la "tecnología" en la función de producción $(F)$, con el valor relativo del bien público en términos de su ratio entre contribución y beneficio para la producción del bien público $(\mathrm{V} / \mathrm{K})$. Según los valores de estas tres variables, podemos obtener unos resultados para $\mathrm{R}, \mathrm{T}, \mathrm{S}$ (ver tabla 1), y con ellos identificar distintas estructuras de juegos clásicos, mediante sus preferencias ordinales (dilema del prisionero, juego del gallina, juego de seguridad, juego del privilegio, dilema del altruista). Además, Heakathron ubica las interacciones basadas en cada uno de estos juegos, en un diagrama donde uno de los ejes es el valor relativo de bien público (V/K), y el otro el exponente tecnológico de la función de producción $(F)$, ambos presentados en escala logarítmica. Con este modelo podemos ver claramente cómo la variable "tecnología de producción" relaciona la contribución al bien con el resultado agregado que se obtiene de éste, y como el valor relativo del bien muestra la relación entre el beneficio que se obtiene y la aportación individual realizada para producirlo.

Las interdependencias señaladas quedan reflejadas en los cinco juegos básicos, con preferencias ordinales, que podemos identificar para cada combinación de R, T y S. Según el tipo de bien, la aportación individual será más o menos recompensada por los beneficios que aporte el bien público (por debajo de 1, el valor relativo del bien público implicaría un retorno negativo a la contribución -lo que implica un juego del altruista en cualquier caso). Por otra parte, la variable $\mathrm{F}$ incide fuertemente en el tipo de interacción posible, ya que para un mismo valor relativo del bien público, superior a 1 , si el valor de $F$ es elevado (mayor de 10), una proporción muy pequeña de todos los consumidores producirá casi todo el bien público posible, y con un valor pequeño de $F$ (menor de 0,1 ) una proporción muy grande de los consumidores podrá producir sólo una proporción pequeña del bien público. En el primer caso nos encontraremos con frecuencia con el juego del gallina y sus problemas de negociación (¿quien hace la contribución?), mientras que en el segundo caso nos encontraremos con el juego de la seguridad y los problemas de coordinación (¿como contribuimos todos?). Por otra parte, existe un espacio en el que $F$ se encuentra en valores cercanos a 1 y el valor relativo del bien toma valores iguales 0 algo superiores a 1, donde encontramos el dilema del prisionero, con todos sus problemas de confianza entre los jugadores para evitar la traición. El dilema del prisionero nos muestra el problema de acción colectiva cuando los beneficios son escasamente superiores a los costes, y la función de producción del bien público es lineal o cercana a la linealidad ( $F=1$ ) -en el fondo, el problema que discutía Olson. Finalmente, otro juego que identifica Heckathorn en su tipología es el juego del grupo privilegiado, que aparece con valores altos del valor relativo del bien público, y en el que no hay conflicto entre racionalidad individual y racionalidad colectiva, ya que contribuir al bien es la mejor opción para todos los jugadores (Heckathorn, 1996: 254-260).

La gran diversidad de bienes públicos que pueden llegar a producirse, y la variación en sus propiedades, complica la aparición de un cierto consenso sobre los parámetros básicos para interpretar la acción colectiva, lo que reafirma la opinión de Ostrom, señalada anteriormente, acerca de necesidad de concebir el análisis de la acción colectiva como 
un campo de estudio, más que una teoría unitaria. Revisando los modelos examinados en esta sección, es posible identificar y distinguir algunas de las estrategias de análisis empleadas. Por una parte, una división importante se encuentra entre los modelos que utilizan variables dicotómicas y los que utilizan variables continuas. Las contribuciones pueden ser continuas (definición de una cantidad) o no (decisión sobre contribuir), por ejemplo, pero también podemos encontrar esta división en la definiciones de los bienes públicos (rivalidad versus no-rivalidad; o bien identificar el grado preciso de rivalidad). Por otra parte, es significativa la estrategia explicativa empleada para incluir las funciones de producción en los modelos. Si en algunos casos sólo se distingue el tipo de bien público y se analiza la dinámica de interacción que genera (Taylor y Ward, 1982, originalmente; Agrawall y Dupont, 1999, como un ejemplo reciente más sofisticado), en otros casos se entra específicamente en la relación entre contribución individual y producción del bien público (Schelling, 1973; Marwell y Oliver, 1993). La propuesta de Heckathorn (1996) destaca especialmente por intentar integrar una cierta variación en la función de producción, identificar tipos distintos de juegos y buscar resultados en términos de relación entre contribuciones, tipo de bien y relación de transformación del bien; no obstante, para ello debe realizar una importante simplificación de todos los parámetros que emplea.

\section{Percepción individual frente a ACCIÓn COLECtiva}

Un aspecto que apenas hemos abordado en este artículo es la formación de las preferencias de los individuos que participan en la acción colectiva, tanto en relación con la producción como con el consumo del bien público. Implícitamente, el uso convencional del supuesto de racionalidad forma parte de los modelos que hemos discutido, junto con las propiedades que identificamos en los bienes públicos. Suponemos que la ordenación de las preferencias de los individuos es consistente y se orienta a obtener el mejor beneficio o utilidad para sí mismos. No obstante, es importante también tener presente que los modelos sobre acción colectiva podrían incorporar otros supuestos sobre el comportamiento de los individuos, que complementen el supuesto de decisión racional, o por el contrario, lo alteren o modifiquen en determinadas condiciones. Un ejemplo de ello lo propone Jones argumentando que la motivación por participar en bienes sin rivalidad puede derivar de su propia naturaleza intrínseca (2004: 459-63). Así, la información sobre la propia naturaleza del bien público puede constituir una señal de bajo coste para reducir los costes de transacción (cálculo de beneficios, obtención de información, control del oportunismo, etc.) que afrontan los individuos en su decisión, estimulando respuestas que vinculen la identidad del bien con la acción colectiva, por una motivación intrínseca, sin calcular costes y beneficios. Jones (2004: 451) destaca el hecho que los individuos generalmente no están tan bien informados como suponen los modelos basados en el comportamiento racional de los actores. Su argumento es que los costes de transacción establecen a menudo un umbral por debajo del cual muchas veces 
los individuos no se detienen a calcular los costes y beneficios de su comportamiento de forma detallada, mientras que las estructuras institucionales sirven como señales de bajo coste para facilitar la acción colectiva.

Un punto de vista distinto sobre el proceso de decisión reside en identificar la forma como se produce el cálculo de costes y beneficios de la participación. Numerosos trabajos se concentran en proponer complejos modelos que reflejen mejor el comportamiento humano que la aplicación directa de un sistema de cálculo matemático simple. Sin entrar a revisar la cuestión, destaca el enfoque de la Prospect Theory, desarrollado por Tversky y Kahneman, que discute en qué medida distintos tipos de situaciones pueden generar de forma estructural y sistemática mayores o menores desviaciones en la percepción individual de las pérdidas y las ganancias. Por tanto, aplicando este enfoque al caso de los bienes públicos, podríamos considerar que cambios sistemáticos en la percepción pueden condicionar los resultados de la acción colectiva, alterando los resultados previsibles, basados en los criterios de racionalidad, sin distorsiones derivadas del contexto en el que se produce la decisión. La aportación más destacada de la Prospect Theory es su observación sistemática de que no existe un comportamiento simétrico de los individuos frente a las pérdidas y las ganancias. Los individuos son más adversos al riesgo de incurrir en pérdidas que favorables a arriesgarse para obtener ganancias, teniendo en cuenta unas condiciones y cantidades similares. Así, en los modelos de acción colectiva, deberíamos poder incorporar que la posibilidad de incurrir en pérdidas generara resistencias a la participación más que proporcionales al valor de las posibles ganancias.

Otra aportación de Tversky y Kahneman son las heurísticas que identifican como formulas de decisión que utilizan los individuos parar enfrentarse a problemas complejos y con elevadores requerimientos de información. Ellos identifican tres heurísticas especialmente importantes. La primera es la heurística de la "representación": inferimos causalidad a partir de observar una cierta similitud entre dos sucesos. La segunda es el mecanismo cognitivo del "anclaje", que se produce cuando una información inicial poco precisa induce a sesgar posteriores juicios sobre la misma cuestión, limitando el alcance de su variación. Finalmente, la tercera heurística es la de la "disponibilidad". Cuanto más visible es un suceso, o más fácil de recordar, es más posible que tengamos tendencia a sobreestimar su frecuencia de ocurrencia (Mercer, 2005: 7-8).

En el análisis de la acción colectiva, la presencia de heurísticas sistemáticas en la toma de decisiones no debe descartarse de ningún modo, ya que puede afectar de forma significativa a la producción del bien público, y explicar la resolución de problemas en casos paradójicos. Las decisiones sobre contribución pueden vincularse a la función de producción del bien público, como hemos visto, así como a su relación con el consumo (el valor relativo del bien público, según Heckathorn). Sin embargo, la percepción del bien público y sus características propias puede incidir de forma destacada en la generación de acción colectiva y la consecuente producción del bien público. Por ejemplo, en la medida que el bien público sea bien visible para los sujetos — tanto en relación con su consumo como con su forma de producción- podemos esperar una mayor tendencia de los individuos a contribuir, por efecto de la heurística de la "disponibilidad". También 
con respecto a la heurística de la "representación", podemos esperar mayor participación en aquellos casos donde la causalidad entre contribución y consumo se perciba de forma más nítida, por la proximidad —en alguna medida— entre lo que se contribuye y lo que se obtiene. Finalmente, con relación a la heurística del "anclaje", podemos pensar en la existencia de individuos cuyas contribuciones no varían de forma simultánea a posibles cambios en las contribuciones de otros, o a cambios en las funciones de producción y sus variables tecnológicas, porque en ambos casos aún siguen tomando como guía el referente inicial.

De los elementos apuntados, podemos observar que la aplicación del enfoque de la Prospect Theory al análisis de los problemas de acción colectiva muestra una significativa potencialidad, así como en general los modelos que realizan un uso más flexible del supuesto de racionalidad, en particular para especificar mejor la relación entre comportamiento individual y los condicionantes del consumo y la producción de los bienes públicos.

\section{Conclusiones}

En las secciones anteriores, hemos planteado distintas aproximaciones al análisis de los problemas de la acción colectiva, tomando siempre como hipótesis que las características y propiedades de los propios bienes públicos podían llegar a explicar en buena medida tales problemas, al establecer unos condicionantes estructurales sobre la lógica de interdependencia de los individuos interesados en disfrutar de tales bienes. Las tipologías y modelos que hemos discutido, con distintos enfoques y criterios, afrontan la necesidad de identificar la complejidad existente, con el fin de generar propuestas de carácter teórico, de aplicación generalizable.

En todo caso, como hemos visto, la idea de un modelo de interacción único para explicar todos los problemas de la acción colectiva puede ser claramente descartada, emergiendo por el contrario la visión de que estos problemas surgen en una gran variedad de situaciones, que requieren de una familia de modelos y variables para interpretarlas y analizarlas sistemáticamente. En todos los casos, en los modelos se encuentran siempre implícitas algunas opciones sobre qué aspectos son más relevantes. Por ejemplo, si se considera que el factor crítico es el propio proceso de producción, parece lógico intentar analizar con detalle la influencia de la tecnología de producción. Por el contrario, si son más relevantes los problemas de rivalidad y desgaste del bien público, los modelos abordan con detalle cómo se produce el consumo del bien. Cuando el factor crítico es la dinámica de interdependencia entre los individuos, se busca simplificar otros aspectos. En este sentido, aunque se tome prioritariamente una posible opción explicativa, debemos considerar que cada modelo permite afinar el análisis de aspectos distintos relacionados con la acción colectiva.

Finalmente, respecto a la inquietud planteada al inicio de este artículo entorno a las dificultades para el avance de la teoría de la acción colectiva, podemos señalar las 
discusiones actuales han introducido mucha más flexibilidad en los debates teóricos, y ya no son frecuentes los grandes enfrentamientos de principios. Sin embargo, también es importante indicar que los problemas más relevantes de la acción colectiva presentan aún múltiples aspectos por esclarecer, dadas las dificultades de contrastar los modelos existentes en numerosos casos. En este artículo hemos explorado sólo unas pocas dimensiones para interpretar la acción colectiva, y ha emergido con rapidez una elevada complejidad en los planteamientos teóricos, sin que conllevaran un debate para ser contrastados empíricamente de forma más sistemática. Ello nos muestra que lo deseable para avanzar en un marco teórico más plausible sobre la acción colectiva sería abordar de forma específica cada uno de sus problemas más significativos, sin pretender una teoría general de la acción colectiva, y comprobar luego de forma específica las propuestas teóricas para tipos de bienes y situaciones particulares de interacción, en distintos planos, tanto empírica como experimentalmente.

\section{REFERENCIAS BibLIOGRÁfICAS}

AGRAWAL, V.K. y C. DUPONT (1999), "Goods, games and Institutions", International Political Science Review, vol. $20, n^{\circ} 4$, pp. 393-409.

AGGARWAL, V.K. y C. DUPONT (2003), "Comment on 'Common Goods, Matrix Games and Institutional Response", European Journal of International Relations, vol. 9, nº 3, pp. 1475-478.

AXELROD, R. (1984), The evolution of Cooperation, Nueva York, Basic Books.

CHAMBERLIN, J. (1974), "Provision of Collective Goods as Function of Group Size", American Political Science Review, vol. 68, n², pp. 707-16.

CORNES, R. y S. TED (1996), The Theory of Externalities, Public Goods and Club Goods, Cambridge, Cambridge University Press.

ESTEBAN, J. y D. RAY (2001), "Collective Action and the Group Size Paradox", American Political Science Review, vol. 95, pp. 663-672.

HARDIN, R. (1971), "Collective Action as an Agreeable N-Prisoners' Dilemma”, Behavioural Science, vol. 16, pp. 472-81.

HECKATHORN, D.D. (1996), "The Dynamics and Dilemmas of Collective Action", American Sociological Review, vol. 61, n² 2, pp. 250-277.

(2002), "Development of a Theory of Collective Action: From the Emergence of Norms to AIDS Prevention and the Analysis of Social Structure", en J. Berger y M. Zelditch (eds), New Directions in Sociological Theory: Growth of Contemporary Theories, San Diego, Rowman and Littlefield.

HESS, C. (2000) (comp.), "Bibliographies from the Workshop Library: Tragedy of the Commons", http://www. indiana.edu/ workshop/ws//tragedy.html. 
HIRSHLEIFER, J. (1983), "From Weakest-Link to Best Shot: the Voluntary Provision of Public Goods", Public Choice, vol. 41, n 3, pp. 371-386.

HOLZINGER, K. (2003), "Reply to Aggarwal and Dupont's Comment to The Editor", European Journal of International Relations, vol. 9, n⿳0 2, pp. 173-212.

HOLZINGER, K. (2003), "Common Goods, Matrix Games and Institutional Response", European Journal of International Relations, vol. 9, n² 2, pp. 173-212.

(2001), "Aggregation Technology of Common Goods and its Strategic Consequences. Global Warming, Biodiversity, and Sitting Conflicts", European Journal of Political Research, vol. 40, pp. 117-38.

JONES, P. (2004), "All for One and One for All': Transactions Cost and Collective Action", Political Studies, vol. 52 , pp. 450-468.

JORDANA, J. (1992), Els limits de la hipotesi de racionalitat com a Jonament per a les teories de l'accio, Barcelona, Publicacions UB.

MARWELL, G. y P. OLIVER (1993), The Critical Mass in Collective Action: A Micro-Social Theory, Cambridge, Cambridge University Press.

MERCER, J. (2005), "Prospect Theory and Political Science", Annual Review of Political Science, vol. 8, pp. $1-21$.

OLIVER, P.E. y G. MARWELL (2001), "Whatever Happened to Critical Mass Theory? A retrospective and Assessment", Sociological Theory, vol. 19, n 3, pp. 292-311.

OLSON, M. (1965), The Logic of Collective Action: Public Goods and the Theory of Groups, Cambridge, MA, Harvard University Press.

OSTROM, E. (1990), Governing the Commons. The Evolution of Institutions for Collective Action, Nueva York, Cambridge University Press.

(2005), Understanding Institutional Diversity, Princeton, NJ, Princeton University Press.

(2003), "How types of goods and property rights jointly affect collective action", Journal of Theoretical Politics, vol. 15, n 3, pp. 239-270.

OSTROM, E., R. GARDNER y J. WALKER (1994), Rules, Games, and Common-Pool Resources, Ann Arbor, University of Michigan Press.

PECORINO, P. y A. TEMINI (2004), "Olson's Logic Revisited: The Role of Small Fixed Costs of Participation", Working Paper Series-Economics, Finance and Legal Studies, University of Alabama.

RAPOPORT, A. (1988), "Experiments with N-Person Social Traps I: Prisoner's Dilemma, Weak Prisoner's Dilemma, Volunteer's Dilemma, and Largest Number", Journal of Conflict Resolution, vol. 32, n 1, pp. 457-472.

RUNGE, F. C. (1984), "Institutions and the Free Rider: The Assurance Problem in Collective Action", Journal of Politics, vol. 46, no 1, pp. 154-181. 
SANDLER, T. (1992), Collective Action. Theory and Applications, Ann Arbor, University of Michigan Press.

SCHELLING, T.C. (1973), "Hockey Helmets, Concealed Weapons and Daylight Savings: A Study of Binary Choices with Externalities", Journal of Conflict Resolution, vol. 17, n 3, pp. 381-428.

SNIDAL, D.J. (1985), "Coordination versus Prisoner's Dilemma: Implications for International Cooperation and Regimes", vol. 79, pp. 923-942.

SZLAGYI, M.N. (2000), "Quantitative Relationships between Collective Action and Prisioners' Dilemma", Systems Research and Behavioural Science, vol. 17, pp. 65-72.

TAYLOR, M. (1987), The Possibility of Cooperation, Nueva York, Cambridge University Press.

TAYLOR, M. y H. WARD (1982), "Chickens, Whales and Lumpy Goods: Alternatives Models of Public Goods Provision", Policy Studies, vol. 30, pp. 350-70. 